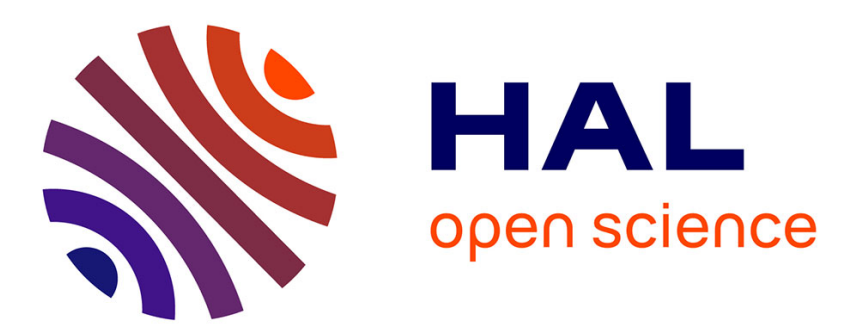

\title{
Insights into dislocation climb efficiency in FCC metals from atomistic simulations
}

\author{
Anas Abu-Odeh, Maeva Cottura, Mark Asta
}

\section{To cite this version:}

Anas Abu-Odeh, Maeva Cottura, Mark Asta. Insights into dislocation climb efficiency in FCC metals from atomistic simulations. Acta Materialia, 2020, 193, pp.172-181. 10.1016/j.actamat.2020.04.047 . hal-03023265

\section{HAL Id: hal-03023265 \\ https://hal.science/hal-03023265}

Submitted on 25 Nov 2020

HAL is a multi-disciplinary open access archive for the deposit and dissemination of scientific research documents, whether they are published or not. The documents may come from teaching and research institutions in France or abroad, or from public or private research centers.
L'archive ouverte pluridisciplinaire HAL, est destinée au dépôt et à la diffusion de documents scientifiques de niveau recherche, publiés ou non, émanant des établissements d'enseignement et de recherche français ou étrangers, des laboratoires publics ou privés. 


\title{
Insights into Dislocation Climb Efficiency in FCC Metals from Atomistic Simulations
}

\author{
Anas Abu-Odeh ${ }^{\mathrm{a}}$, Maeva Cottura ${ }^{\mathrm{a}, \mathrm{b}}$, Mark Asta $^{\mathrm{a}, \mathrm{c}, *}$ \\ ${ }^{a}$ Department of Materials Science and Engineering, University of California, Berkeley, CA 94720, USA \\ ${ }^{b}$ Institut Jean Lamour, CNRS - Université de Lorraine, Nancy F-54011, France \\ ${ }^{c}$ Materials Sciences Division, Lawrence Berkeley National Laboratory, Berkeley, CA 94720, USA
}

\begin{abstract}
Dislocation climb is a mechanism fundamental to high temperature deformation processes, annealing of quenched-in defects, and irradiated defect absorption in metals. It is known from experimental observations and theoretical considerations that climb velocities in face-centered-cubic (FCC) metals can be slower than expected from diffusion kinetics, due to limited jog availability. Here we investigate the so-called climb efficiency, i.e., the ratio of the steady state climb velocity to that expected from diffusion-limited rates, employing atomistic simulations for FCC metals within the framework of a previously developed analytical model. The simulations consider the energetics of jog-pair formation and vacancy migration in the vicinities of edge-dislocations in $\mathrm{Al}$ and $\mathrm{Cu}$. Calculated climb efficiencies for the lower-stacking-fault-energy $\mathrm{Cu}$ system are found to be substantially lower than those for $\mathrm{Al}$ over a wide range of homologous temperatures. These results agree qualitatively with experimental observations showing that the dislocation climb efficiency in FCC materials tends to be lower in systems with smaller stacking fault energy. Considerably higher energies and more complex kinetic pathways are reported for jog-pair formation in $\mathrm{Cu}$ than in $\mathrm{Al}$, which correlates with the larger dissociation distance between partial dislocations in this system. This work highlights how atomistic modeling can be used to enhance mesoscale models of climb, and suggests alloy design considerations to tune climb efficiencies.
\end{abstract}

Keywords: edge dislocation, climb, face-centered cubic crystals, creep, irradiated metals

\section{Introduction}

In the plastic deformation of crystals, climb is a process that enables dislocations to overcome obstacles in a slip plane through the absorption or emission of vacancies. Driving forces for climb arise due to supersaturation of point defects and elastic stress, providing a mechanism to evolve the system towards equilibrium. Dislocation climb under both types of driving forces is influenced by the rate of arrival of vacancies, and when their flux is the rate-limiting factor, analytical theories can be developed for steady-state climb velocities. However, it has long been appreciated

\footnotetext{
${ }^{*}$ Corresponding author

Email address: mdasta@berkeley.edu (Mark Asta)
} 
[1-3] that limited availability of jogs along a dislocation line can lead to a reduction in climb velocity relative to this diffusion-limited value. As reviewed in the next section, the notion of a climb efficiency, defined as the ratio of the actual climb velocity to the ideal diffusion-limited value [1], has been introduced to quantify such deviations.

Climb rates in different FCC metals with quenched-in vacancy loops have been determined by transmission electron microscopy through the measurement of the shrinkage kinetics under annealing conditions, which are related to the vacancy emission rate [4, 5]. These experiments have shown that in FCC metals, there is a trend for lower stacking fault energy (SFE) metals to have lower climb efficiencies. Other less direct experiments, which estimate the driving forces for climb based on quenching conditions and mechanical stresses, lead to similar conclusions [6]. While there is consensus on this qualitative trend related to the decrease in climb efficiency with decreasing stacking fault energy, quantitative relations between driving force and climb efficiency that can be compared to available theoretical models (see below) are challenging to derive directly from currently available experimental data. This is due to the presence of combined contributions to the driving force arising from curvature, external stress, and vacancy supersaturation, and the possible role of a combination of different diffusion mechanisms. For example, detailed measurements and analyses of vacancy loop growth in FCC Al [6, 7] led to estimates for climb efficiency assuming growth due to the absorption of vacancies emitted by smaller loops. However later studies proposed a mechanism where a vacancy loop could migrate through "self-climb" as a consequence of short-circuit diffusion in the dislocation core [8, 9] and the effect of this mechanism on the climb efficiency estimates has not been undertaken. Climb efficiencies were also estimated based on experiments for FCC Au displaying dislocation networks [10]; these microstructures displayed stacking fault tetrahedra (SFT), which can have diffusivities that are orders of magnitude faster than mono-vacancies [11] and the effect of this transport mechanism on the estimated efficiencies remains unclear. In light of the these types of complicating factors characteristic of realistic microstructures, mesoscale and atomistic simulation methods provide a complementary framework for derive fundamental relations between driving forces and climb rates.

Mesoscale methods such as discrete dislocation dynamics (DDD) and phase field models (PFM) offer an attractive framework to model dislocation climb processes. However, for such applications, models and associated assumptions have to include the relative contributions of vacancy diffusion and "attachment" processes associated with the concentration of jogs and vacancy attachment to them. A number of DDD models assume diffusion-limited climb rates, where barriers for attachment of vacancies to the dislocation are considered unimportant [12,-14], which is suitable for metals with high climb efficiencies. By contrast, the model introduced by Gao et al. assumes attachment-limited kinetics suitable for a low-jog density along the dislocation line [15]. Additional DDD models have introduced parameters to describe the effects of non-ideal climb efficiencies [16, 17] whose values must be derived from microscopic theories. PFM implementations provide an alternative framework for modeling dislocation climb processes. The PFM models introduced by Ke et al. [18] and that of Geslin et al. [19, 20] allow for mixed attachment and diffusion-limited behavior through the introduction of an attachment kinetics parameter that must be fit to experiments or atomistic 
simulations. Liu et al. illustrated the importance of accurately describing the mixed kinetics of climb in a PFM of low-angle grain boundaries where, depending on the kinetics of vacancy attachment, the variation of grain boundary sink strength can influence void nucleation under vacancy supersaturation [21]. Gu et al. also demonstrated that a deviation from a dislocation being a perfect sink/source of vacancies can change the self-healing time of perturbed low-angle grain boundaries [22].

To enhance predictive capabilities in the modeling of dislocation climb, information about vacancy and jog properties can be derived from calculations at the atomic scale. Relevant atomistic computational work can be summarized as follows. Density-functional theory (DFT) calculations on the effect of solutes on the SFE in Ni and vacancy diffusion effects on climb were carried out by Yu and Wang [23], assuming that the energy of the jog is governed by the cost of constricting the two Shockley partials, which is believed to provide an upper bound [3]. Sarkar et al. used diffusive molecular dynamics (MD) to study the evolution of a jog-pair in FCC Cu [24], and a displacive-diffusive path associated with climb was identified. In further atomistic studies, Baker and Curtin employed a mixture of accelerated MD simulations and solutions to a discrete diffusion equation to investigate jog-pair formation in FCC Al [25]. Lau et $a l$. investigated the jog structure and vacancy attachment energies for a mixed dislocation in BCC Fe using the nudged elastic band (NEB) method [26]. Using the energies from this study, Kabir et al. parameterized a kinetic Monte Carlo (kMC) model to simulate climb velocity under a high supersaturation of vacancies and to extrapolate creep behavior [27]. It was shown that an analytical equation of climb matched these results, although the high dislocation densities in these simulations complicate extrapolation to conditions more typical of creep experiments [28]. Swinburne et al. parameterized a kMC model to study the significant effects of self-climb versus vacancy mediated climb on interstitial loops in Fe and $\mathrm{W}$, and the effects are postulated to be of the same magnitude for FCC materials [9]. These studies demonstrate the insights into climb behavior that can be derived from atomistic simulations, and the use of quantities calculated at this scale in mesoscale models.

To build on the modeling work summarized above, this study employs atomistic simulations to investigate the energetics underlying jog formation and vacancy attachment for representative high and low stacking fault FCC metals, $\mathrm{Al}$ and $\mathrm{Cu}$, respectively. The energetics obtained from these simulations are used to understand consequences for climb efficiencies within the kinetic theory of climb developed by Balluffi [1]. In addition, energetic barriers associated with constriction of vacancy aggregates into jog-pairs and vacancy diffusion towards extended jogs are explored using the NEB method [29, 30]. To motivate the atomistic calculations undertaken in this work, Section $\$ 2$ presents a summary of the dislocation climb theory due to Balluffi [1]. Section $\S 3$ describes the simulation setup and computational details. Results for jog-pair formation energetics and the change in vacancy migration barriers near a jog are presented in Section $\S 4$, and Section $§ 5$ applies Balluffi's climb theory to quantify climb efficiencies in $\mathrm{Al}$ compared to $\mathrm{Cu}$. Section $\S 6$ provides a summary of the main conclusions from this work. 


\section{Dislocation Climb Theory}

In this section we review the theoretical model for dislocation climb due to Balluffi [1], which motivates the atomistic calculations described in the next section. The model considers dislocation climb under vacancy supersaturation driving forces, and involves six main assumptions:

1. the dislocation is straight, unconstrained, and is in a low index direction

2. jogs nucleate homogeneously by vacancy aggregation along the core

3. vacancies reaching the core diffuse rapidly to the jogs

4. the dislocation is undissociated

5. vacancy quasi-equilibrium is maintained at the jogs

6. the energy of embryonic jog pairs increases monotonically with size

The model considers the diffusion of vacancies to jogs, which are treated as ellipsoidal sinks situated at an equilibrium spacing along the dislocation line (Figure 11). The solution to the diffusion equation provides a steady-state velocity $\left(v_{\text {mixed }}\right)$ in the so-called "mixed" kinetic regime given by:

$$
v_{\text {mixed }}=2 \pi D b^{2} \frac{2 Z}{\lambda} \frac{\left[c(R)-c^{0}\right]}{\left[\ln \left(\frac{2 Z}{\lambda} \frac{\lambda}{b}\right)+\frac{2 Z}{\lambda} \ln \left(\frac{R}{\lambda}\right)\right]}
$$

where $c(R)$ is the supersaturated concentration of vacancies in the bulk material at a distance $\mathrm{R}$ far from the jog. $c^{0}$ is the vacancy concentration in equilibrium with the jogs, taken to be the equilibrium bulk vacancy concentration consistent with the assumption of local thermodynamic equilibrium. $D$ represents the bulk vacancy diffusivity and $b$ is the Burgers vector. The mean-free path of a vacancy in the dislocation core $(Z)$ is given by:

$$
Z=\sqrt{2} b \exp \left(\frac{\Delta W_{s m}}{2 k T}\right)
$$

where $\Delta W_{s m}=E_{b}-E_{c}$ is the difference between the self-migration energy, defined as the summation of the formation energy and the migration energy, of a vacancy in the bulk $\left(E_{b}\right)$ and the core $\left(E_{c}\right) . k$ is the Boltzmann constant and $T$ is the absolute temperature. The equilibrium distance between jogs $(\lambda)$ along the dislocation is given by the expression:

$$
\lambda=b \exp \left(\frac{\Delta F_{\text {jogpair }}^{*}}{2 k T}\right)
$$

where $\Delta F_{\text {jogpair }}^{*}$ is the free energy change associated with jog-pair formation. This free energy change is multiplied by $1 / 2$ because it is assumed that each jog contributes equally to the jog-pair formation energy. In this model, the thermodynamic driving force for dislocation climb is given by the difference in vacancy chemical potential $(\Delta \mu)$ at the 
jogs versus in the far field. In the dilute limit, this value is given by:

$$
\Delta \mu=k T \ln \left(\frac{c(R)}{c^{0}}\right)
$$

In applying this theory, $R$ is taken as half the value of the average dislocation spacing:

$$
R=\frac{1}{2} \rho^{-\frac{1}{2}}
$$

where $\rho$ is the dislocation density.

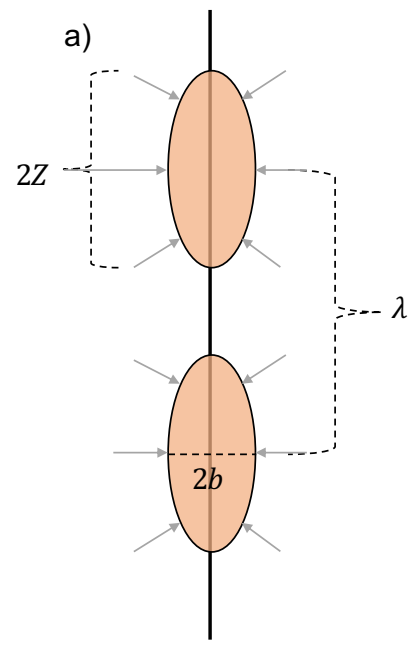

b)

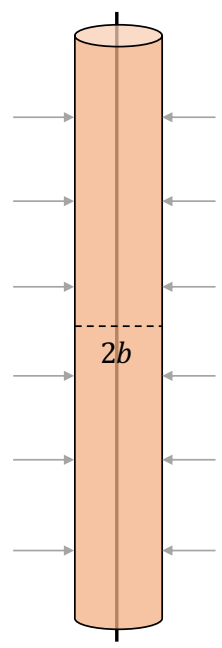

Figure 1: Representation of vacancy sinks when a) $\frac{2 Z}{\lambda}<1$ and when b) $\frac{2 Z}{\lambda} \geq 1$. Vacancies fluxes are schematically represented by the gray arrows.

The critical condition of $\frac{2 Z}{\lambda}=1$ represents the overlapping of the ellipsoidal sinks (see Fig. 1), and in this limit Eq. (1) becomes:

$$
v_{\text {diff-control }}=2 \pi D b^{2} \frac{\left[c(R)-c^{0}\right]}{\ln (R / b)},
$$

which represents the diffusion controlled limit of the climb velocity. The actual climb velocity $\left(v_{\text {actual }}\right)$ depends on the ratio of $Z$ to $\lambda$, and is given by:

$$
v_{\text {actual }}= \begin{cases}v_{\text {mixed }}, & \text { if } \frac{2 Z}{\lambda}<1 . \\ v_{\text {diff-control }}, & \text { otherwise. }\end{cases}
$$

The climb efficiency, $\eta$, is then defined as:

$$
\eta=\frac{v_{\text {actual }}}{v_{\text {diff-control }}}
$$

and provides a measure of the reduction in climb velocity relative to the idealized diffusion-limited case.

The limiting case of $\eta=1$ corresponds to ideal diffusion-limited climb velocities, while values of $\eta<1$ indicate 
the importance of attachment processes at the jogs in limiting the climb velocity. Mesoscale models of climb that incorporate mixed kinetics are dependent on ratios similar to $Z / \lambda[16,17]$ or are dependent on $\lambda$ [20]. These values are difficult to derive directly from experimental measurements, and an alternative is to determine them from simulations at the atomic scale. The next section discusses the applications of atomistic simulations for FCC metals in this context.

\section{Simulation Details} $\mathrm{Cu}$ are converged with respect to system size to within approximately $14 \%$. Details of this analysis are given in the Supplementary Document.

Using the simulation cells described above, the energy of embryonic jog pairs were investigated using two alternative algorithms as presented in Section \$4.1. In the first, which we will refer to as "non-annealed", an atom with 


\begin{tabular}{|c|c|c|c|c|}
\hline Material & $C_{11}(\mathrm{GPa})$ & $C_{12}(\mathrm{GPa})$ & $C_{44}(\mathrm{GPa})$ & $a_{\text {lat }}(\AA)$ \\
\hline $\mathrm{Al}$ & 110 & 61 & 33 & 4.045 \\
\hline $\mathrm{Cu}$ & 175 & 128 & 84 & 3.639 \\
\hline
\end{tabular}

Table 1: Elastic constants and lattice constants for the EAM potentials of $\mathrm{Al}$ and $\mathrm{Cu}$ [37] used in the atomistic simulations.
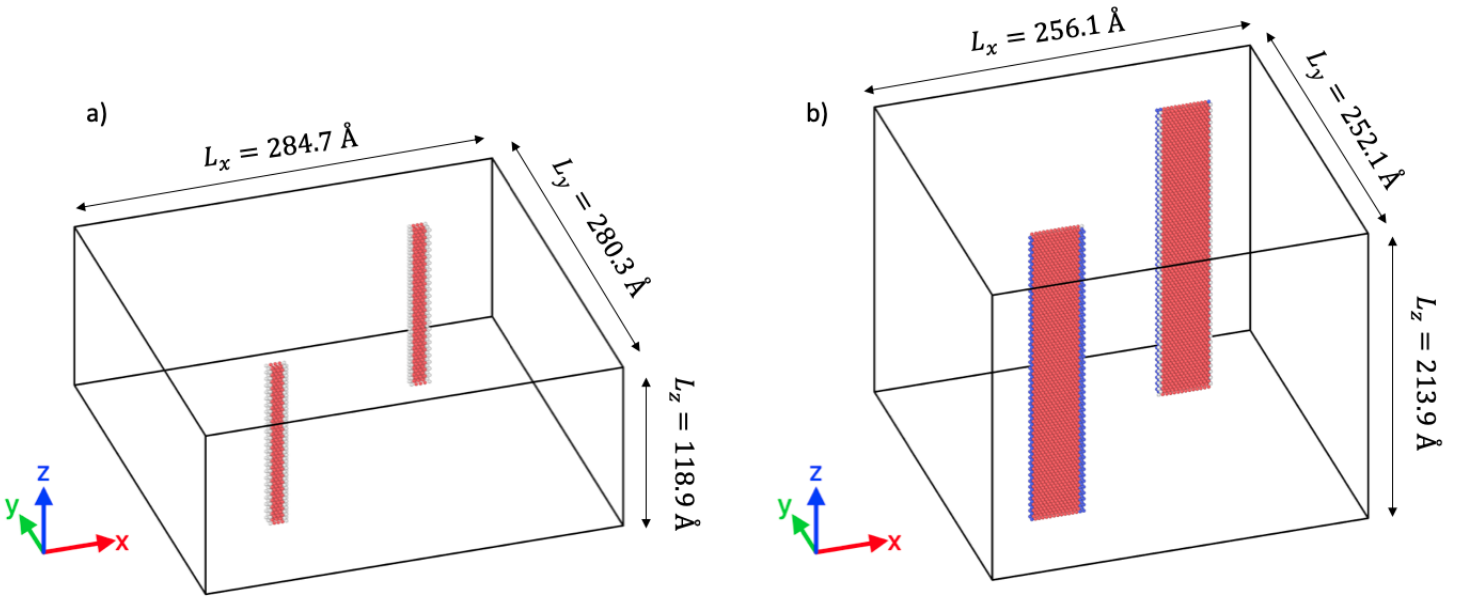

Figure 2: Simulation box containing two edge dislocations after relaxation for a) $\mathrm{Al}$ and b) $\mathrm{Cu}$. Atoms in FCC environments were deleted according to the common neighbor analysis performed with OVITO [40 41]. Red atoms represent the stacking fault region, and the remaining atoms represent the partial dislocations.

the highest potential energy in the compressive region of the dislocation near one of the two partials of an otherwise perfect dissociated dislocation is deleted to form a vacancy. The procedure is similar to the one applied by Dos Reis $e t$ al. [42] for advancing a constricted jog on a dislocation with a nanovoid. After introducing the vacancy, the simulation cell was relaxed using FIRE minimization with a force convergence of $0.001 \mathrm{eV} / \AA$. The atomic sites on the rows above and below (with respect to the z-axis of the simulation cell) the original vacancy on the dislocation core are searched to find the next minimum energy vacancy site and the corresponding atom is deleted, followed by a FIRE relaxation with a convergence of $0.001 \mathrm{eV} / \AA$. The process is repeated, searching atomic rows directly above and below the vacancy aggregation until the desired number of vacancies have been absorbed by the dislocation. The resulting energy, $E_{\text {jog pair }}$, of the defect introduced by the vacancies is given by:

$$
E_{\text {jogpair }}=E_{1}-N_{1} * E_{c o h}-\left(E_{2}-N_{2} * E_{c o h}\right)
$$

where $E_{1}$ is the total energy of the cell with the dislocations and the added vacancies, $N_{1}$ is the number of atoms in that cell, $E_{2}$ is the total energy of the cell with just the dislocations, $N_{2}$ is the number of atoms in that cell, and $E_{c o h}$ is the cohesive energy per atom, which for FCC Al is $-3.4107 \mathrm{eV} /$ atom, and for FCC Cu is $-3.2831 \mathrm{eV} /$ atom, with the interatomic potential models employed in these calculations. The quantity $E_{\text {jogpair }}$ defined in Eq. (9) allows for the 
calculation of the critical free energy change needed in Eq. 33, which is derived as the maximum of the quantity:

$$
\Delta F_{\text {jogpair }}\left(N_{\text {vacs }}\right)=E_{\text {jogpair }}\left(N_{\text {vacs }}\right)-N_{\text {vacs }} * \Delta \mu
$$

where $N_{\text {vacs }}$ is the number of vacancies absorbed by the dislocation, and $\Delta \mu$ is the chemical potential driving force relative to the non-annealed structures obtained from the vacancy-absorption and energy minimization process. The energy difference between annealed and non-annealed structure was most pronounced for $\mathrm{Cu}$, as discussed in Section $\$ 4.1$ The energy barrier associated with the transformation from the non-annealed to annealed structure for $\mathrm{Cu}$ was investigated using the NEB [29, 30] method. Such NEB calculations were performed for four annealed and nonannealed jog structures at various values of absorbed vacancies. Due to a sharp transition point in the minimum energy path, 48 images were used between the initial (non-annealed) and final (annealed) state. Afterwards, two more iterations of NEB each with 48 images were carried out, with the initial and final state taken as fixed images within 
the minimum-energy pathway derived from the previous iteration, in order to increase the density of images near the saddle point. Energy versus reaction coordinate plots and convergence of energy barriers with NEB iterations are given in the Supplementary Document. These calculations were performed with a convergence criteria of $0.01 \mathrm{eV} / \AA$.

The NEB method was also employed for the calculated results presented in $\$ 4.2$ to investigate vacancy migration barriers near a jog. In these simulations, a jog-pair structure was created on a dislocation with a jog spacing of half of the simulation cell height. This was done by deleting atoms on the extra half plane associated with the edge dislocation before relaxing the dislocations as outlined above. For extended and constricted jogs in both $\mathrm{Al}$ and $\mathrm{Cu}$, a series of climbing image NEB [29, 30] calculations were performed of a vacancy following a tensile, compressive, or side path to the jog based on a chain of 5 closed-packed jumps in the desired direction as illustrated in Fig. 6 The force convergence used for the tensile and compressive paths was $0.01 \mathrm{eV} / \AA$ with 5 images used for each jump. The side paths were relaxed to a convergence of $0.03 \mathrm{eV} / \AA$. This was done to avoid convergence issues due to the dislocation bowing out due to its attraction to the vacancy. This gives an upper bound of the jump barriers because as the dislocation bows towards the vacancy, some of the barriers in the path will decrease or disappear.

\section{Simulation Results}

In this section, we describe the results of the atomistic simulations in the context of the theoretical model reviewed in Section \$2 . Beforehand, we review the underlying assumptions of this model. First, the simulation geometries are designed to consider straight edge dislocations in an otherwise perfect crystal, such that they correspond to the assumptions 1 and 2 of the theoretical model. They are also designed to be consistent with assumption 3, which follows the idea that dislocation core diffusion is generally vacancy mediated. While molecular dynamics studies of vacancy and interstitial diffusivities in edge dislocations in $\mathrm{Al}[43]$ and $\mathrm{Cu}$ [44] show that this is valid in $\mathrm{Al}$, for $\mathrm{Cu}$ both the interstitial and vacancy mechanism have been found to contribute equally. However, since our intent in this study, consistent with the theoretical model, is to study climb under vacancy supersaturation conditions, assumption 3 is viewed to be reasonable. Assumption 4, which states that the dislocations are not dissociated, is generally not true for FCC materials. However, this should not present a problem for the analysis below since the effects of dissociation are inherently included in the calculations of jog-pair formation energy. Assumption 5 is satisfied if it can be shown that the barrier for each jump of a vacancy from the bulk to the jog is never larger than the bulk migration barrier, and that the barriers to the jog are appreciably smaller than the reverse path [3]. Assumption 5 would break down if a high-energy barrier prevents the vacancy from easily being absorbed by an extended jog, e.g., it would require the partial dislocations to constrict first. We will investigate this issue using results of NEB calculations in Section $\$ 4.2$. and show that the assumption holds for the present cases studied. Finally, assumption 6 is valid if there is no highenergy vacancy complex that forms before the nucleation of a jog-pair. This is explored by using a mix of molecular 
statics and simulated thermal annealing in $\$ 4.1$

We note that the Balluffi model neglects explicit incorporation of the interactions of the stress field of a dislocation with vacancies. These interactions will change the equilibrium vacancy concentrations near the dislocation as well as the mobilities underlying vacancy diffusion. PFM results [18, 20] for dislocation climb that have considered such elastic interaction effects suggest that they have a relatively small effect on the resulting climb velocity, especially when no applied stress is present. Thus, in the application of the Balluffi model to investigate climb efficiency these effects will not be considered further in what follows.

\subsection{Structure and Energetics of Jogs}

Figure 3 plots formation energies of dislocations with jogs and vacancy clusters as a function of the number of absorbed vacancies, with insets illustrating several representative structures. Results are presented for both nonannealed (green for $\mathrm{Cu}$ and blue for $\mathrm{Al}$ ) and annealed (red for $\mathrm{Cu}$ and orange for $\mathrm{Al}$ ) structures. The annealing search results in lower jog-pair formation energies, particularly for $\mathrm{Cu}$, as the thermal fluctuations are able to overcome the barrier associated with constricting the vacancy clusters across the partials. Considering first the results for $\mathrm{Cu}$, it is seen from the inset that the absorption of up to ten vacancies leads to the formation of an extended vacancy cluster centered on one of the two partial dislocations in the non-annealed samples. In these non-annealed samples the formation energy and area of these vacancy clusters increases monotonically until 32 vacancies are absorbed, at which point the vacancy-cluster structure collapses to form a jog-pair structure illustrated by the bottom right inset. This latter structure is consistent with the idea of a "well-formed" jog-pair structure in the theoretical model of Balluffi [1]. This jog-pair structure features one compact constricted jog (top) and one extended jog (bottom), with bowing of the partial dislocation line segments in between. Further addition of vacancies causes the jog pairs to separate but with the structure of the individual jogs remaining qualitatively similar. The annealed samples for $\mathrm{Cu}$ give the same structure as the non-annealed samples for up to ten absorbed vacancies. Beyond that, annealing leads to the formation of the constricted/extended jog-pair structure described above. In the annealed samples there is a slight increase in formation energy beyond approximately 11 absorbed vacancies, due to the decreasing effects of the jog-jog interaction as the jogs spread further apart. The distance between the jog-pairs is never larger than half of the supercell height in the simulations, to minimize the jog interactions with their periodic images.The jog-pair formation energies level out to a value of approximately $6.5 \mathrm{eV}$ beyond approximately 35 absorbed vacancies. We note that the dissociated vacancy cluster structure found here for the non-annealed samples is similar in nature to that obtained by Sarkar et al. [24], who also identified an energetically downhill path between this cluster structure and the jog-pair structure in $\mathrm{Cu}$.

For $\mathrm{Al}$, the results of the structures as a function of absorbed vacancies are qualitatively similar to those for $\mathrm{Cu}$, with the main differences being associated with the much narrower width of the dissociated dislocation core. Specifically, the extended vacancy cluster type of structure (see blue framed inset) persists up to four (three) absorbed vacancies 


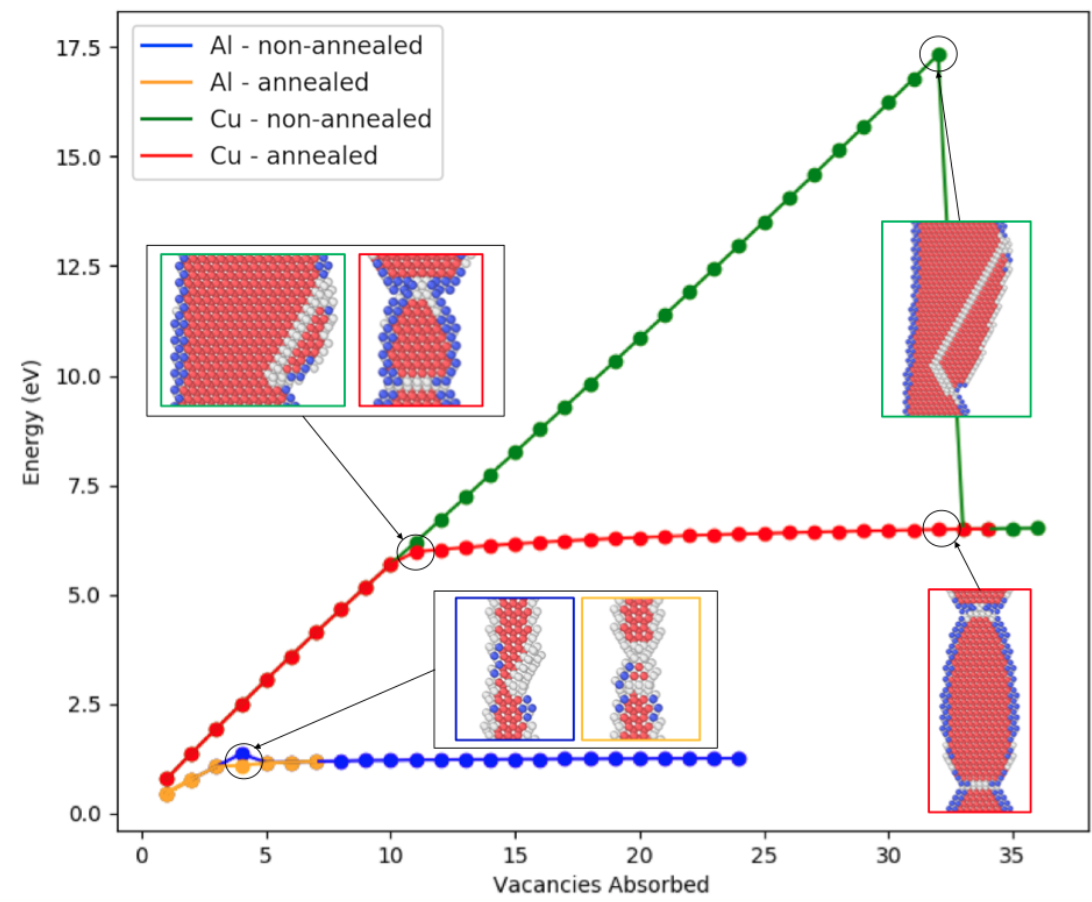

Figure 3: Jog-pair formation energy as a function of the number of vacancies absorbed for $\mathrm{Al}$ (up to 24) and $\mathrm{Cu}$ (up to 36). Insets show the change of the dislocation structure with and without simulated annealing, with the color of the border of the dislocation images corresponding to the color of the energy path. The dislocations were colored using the common neighbor analysis (CNA) scheme in OVITO [40 41]. FCC type atoms were removed, red atoms represent an HCP type environment, and blue and grey represent atoms that belong to the partial dislocations or the jogs.

in the non-annealed (annealed) samples. Beyond that the jog-pair structure with a constricted and extended jog forms and addition of more absorbed vacancies leads to an increase in the distance between the jogs, with the structure of the jogs remaining qualitatively similar. The formation energies are uniformly much lower for $\mathrm{Al}$ than for $\mathrm{Cu}$ for all values of the number of absorbed vacancies. For Al, the formation energy plateaus at a value of approximately $1.3 \mathrm{eV}$.

The results for the annealed samples in Fig. 3 point towards the satisfaction of assumption 6 in the model of Balluffi, namely that the energy of embryonic jog pairs increases monotonically with size without the presence of an appreciable extra energy barrier to form well-formed jog pairs. However, it should be noted that for the case of the annealed sample of $\mathrm{Cu}$ with eleven absorbed vacancies, the constricted/extended jog-pair structure is not always found within the length of the annealing simulations using different random number seeds for the velocity distributions. This hints at the presence of an appreciable activation energy barrier from between the non-annealed and annealed structures in $\mathrm{Cu}$ with a decreasing number of vacancies absorbed. The energy barriers between selected pairs of annealed and non-annealed structures for $\mathrm{Cu}$ were thus computed from NEB calculations with results plotted in Fig. 4. The transition states for these configurations all show a partial constricting to meet the vacancy cluster, which is a different mechanism for jog-pair formation than that suggested by Sarkar [24] and Grilhé [45] where the vacancy cluster dissociates across the stacking fault ribbon.

The results in Fig. 4 show the trend of a decreasing energy barrier with increasing number of vacancies absorbed 
to transform from non-annealed to annealed structures in $\mathrm{Cu}$. This trend is qualitatively consistent with previous calculations based on elasticity theory by Grilhé et al. [45]. The lower activation barriers for the larger vacancy aggregates can be understood to arise since in these structures dissociating into jog pairs does not require constricting

the partials as much as in the structures with smaller vacancy aggregates. The results imply that there could be different kinetic pathways for formation of jogs in $\mathrm{Cu}$ depending on the temperature, where the system follows the non-annealed energetics (the green curve in Fig. 3 until the barrier to transform to the annealed structure (the red curve in Fig. 3 ) is low enough to be thermally activated. These different kinetic pathways could explore different directions in the displacive-diffusive space described by Sarkar et al. [24]. Also, it is assumed here that the vacancy cluster only spans one climb plane. With low SFE materials such as $\mathrm{Cu}$, there is a possibility that jogs that span more than one climb plane could form, which would be expected to lead to more complex kinetic pathways. To properly quantify the different paths, the minimization search would need to span more than just one plane, and a large sampling size of simulated annealing attempts would be required in order to explore the lower energy structures. Afterwards, transition barriers would need to be computed between these states to find the likelihood of observing one of those states. Another strategy for finding jog structures in low SFE materials would be to adapt the procedure of Grilhé at al. [45] in molecular statics to find the vacancy cluster of an appropriate dimension which would lead to a spontaneous jog-pair formation. These strategies are beyond the scope of the present work, and the given results are taken as a satisfactory approximation since the simulated annealing of most of the vacancy clusters readily yield a jog-pair structure on one climb plane. A further complication to the kinetic pathway evaluation of low SFE materials is including paths where a vacancy might attach at a less energetically favorable site, such as the partial opposite of the vacancy aggregate or in the middle of the stacking fault. In the analysis of Section $\$ 5$, the energetic pathway identified here is assumed to be the lowest energy one present for both $\mathrm{Al}$ and $\mathrm{Cu}$.

\subsection{Vacancy Hopping Barriers}

As described above, one of the assumptions of the Balluffi model (assumption number 5 listed in Section $\$ 2$ ) would break down if a high energy barrier exists for a lattice vacancy to be absorbed at a jog. In this sub-section, we investigate this issue employing NEB calculations to obtain energy barriers for vacancy hopping to the jog structures shown in Fig. 5 (a) and (b) for $\mathrm{Al}$ and $\mathrm{Cu}$, respectively. For both the extended and constricted jogs in $\mathrm{Al}$ and $\mathrm{Cu}, \mathrm{a}$ series of climbing image NEB calculations were performed of a vacancy following a tensile, compressive, or side path to the jog based on a chain of 5 closed-packed jumps in the desired direction. Figure 6 shows example paths for the extended jog in $\mathrm{Cu}$.

The results of the NEB calculations are shown in Fig. 7. The zero energy value is in reference to the simulation cell without an inserted vacancy. A comparison of the compressive and tensile paths shows that the minima in the tensile paths (for jump \#0: approximately $1.080 \mathrm{eV}$ and $1.091 \mathrm{eV}$ for the $\mathrm{Cu}$ extended and constricted paths, respec- 


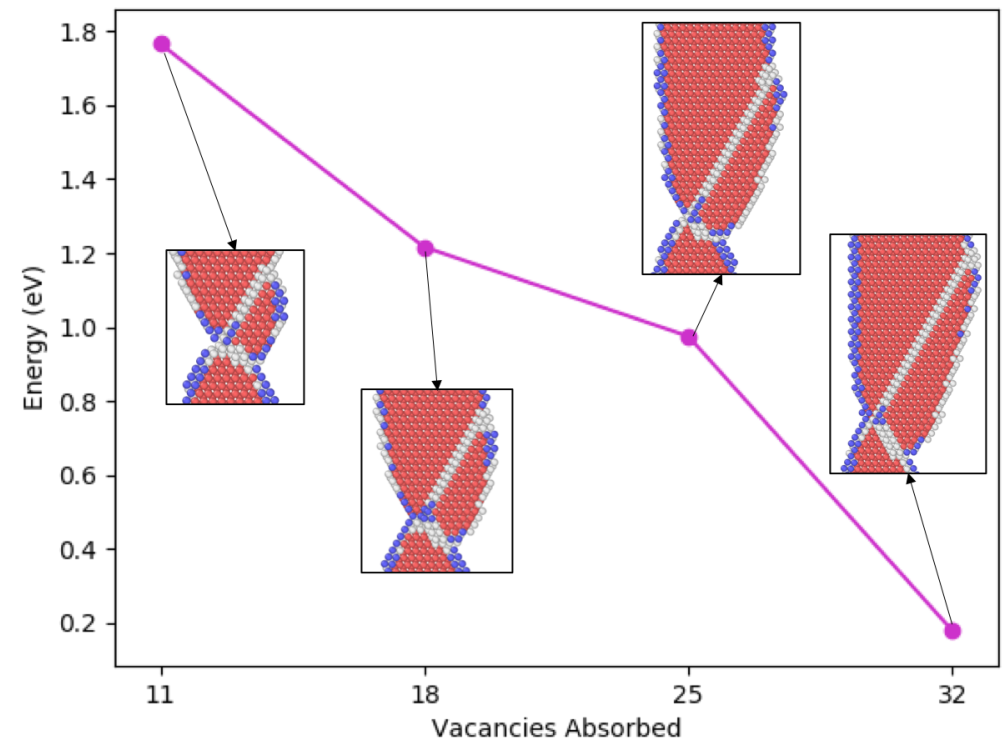

Figure 4: NEB barriers for $\mathrm{Cu}$ jog-pairs between non-annealed to annealed states. Insets show the structure of the transition state between the vacancy cluster and the jog-pair.

tively, and approximately $0.708 \mathrm{eV}$ and $0.712 \mathrm{eV}$ for the $\mathrm{Al}$ extended and constricted paths, respectively) before the destruction of the vacancy is higher than those in the compressive paths (for jump \#0: approximately $1.013 \mathrm{eV}$ for the $\mathrm{Cu}$ extended and constricted paths, and approximately $0.615 \mathrm{eV}$ and $0.614 \mathrm{eV}$ for the $\mathrm{Al}$ extended and constricted paths, respectively). This is expected because vacancies are attracted to regions of compression. The final jump of the vacancy to the jog (rightmost point in the figures) for all of the paths in both materials have either the lowest migration barrier or no barrier. This indicates that high-energy constriction of partial dislocations in $\mathrm{Cu}$ is not necessary for a vacancy to annihilate at an extended jog. Instead, the vacancy advances the jog by dissociating into an extended jog, similar to the "1/3" vacancy mechanism discussed in [1, 46]. Note that the energy difference between the vacancy at position 0 and at position 5 for all the paths correspond to the value of the vacancy formation energy (where the bulk value is calculated to be $0.658 \mathrm{eV}$ for $\mathrm{Al}$ and $1.048 \mathrm{eV}$ for $\mathrm{Cu}$ from the respective potentials), indicating that the vacancy is annihilated from the system.

The bulk vacancy migration energy was calculated using the NEB method for a vacancy in an otherwise perfect cell with a force convergence of $0.001 \mathrm{eV} / \AA$. The values were found to be $0.638 \mathrm{eV}$ for $\mathrm{Al}$ and $0.988 \mathrm{eV}$ for $\mathrm{Cu}$, with the EAM potentials employed in this work. By inspection, all of the side paths seem to fit assumption 5 as they do not have barriers larger than the bulk migration energy and they are decreasing in the direction from the bulk to the jog (and thus increasing in the reverse direction). A few exceptions exist for the compressive and tensile paths (indicated by the barriers in Fig. 7 above the black line representing the bulk self-migration energy in the material), but the differences with the bulk migration energy are small compared to the activation barrier. The NEB calculations in [26] 


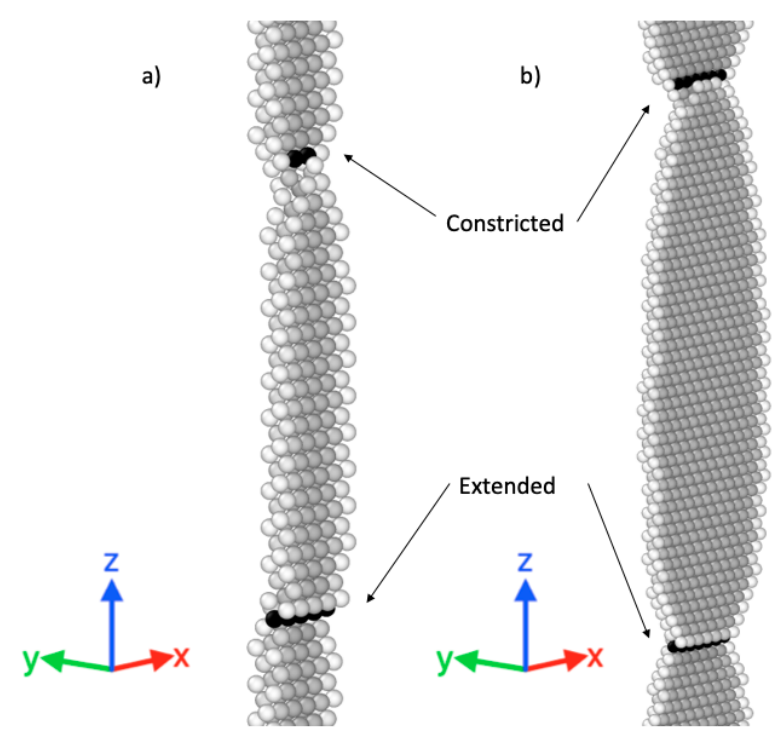

Figure 5: Jog structure used for NEB simulations for a) $\mathrm{Al}$ and b) $\mathrm{Cu}$. Black atoms represent regions where absorbing a vacancy will allow a jog to migrate one atomic row.

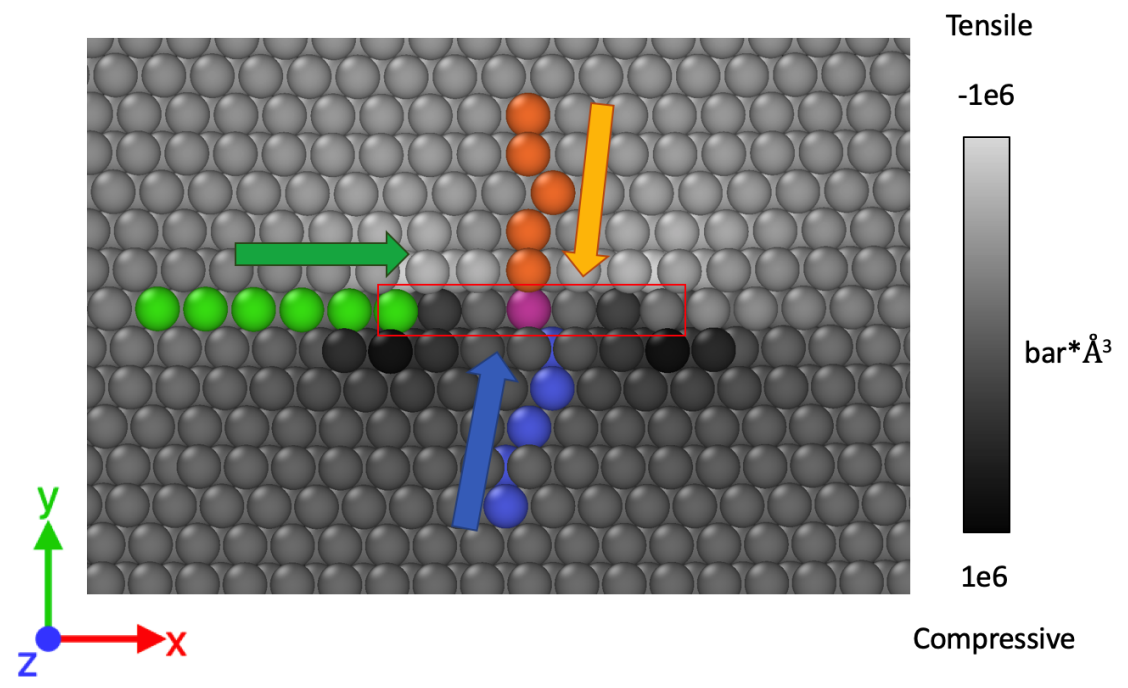

Figure 6: NEB vacancy paths for an extended jog in Cu looking down the z-axis. The color bar represents the hydrostatic pressure on each atom output from LAMMPS [39]. Orange atoms represent a tensile path, blue atoms represent a compressive path, and green atoms represent a side path. The red box represents the black atoms for the extended jog in $\mathrm{Cu}$ from Fig. 5 

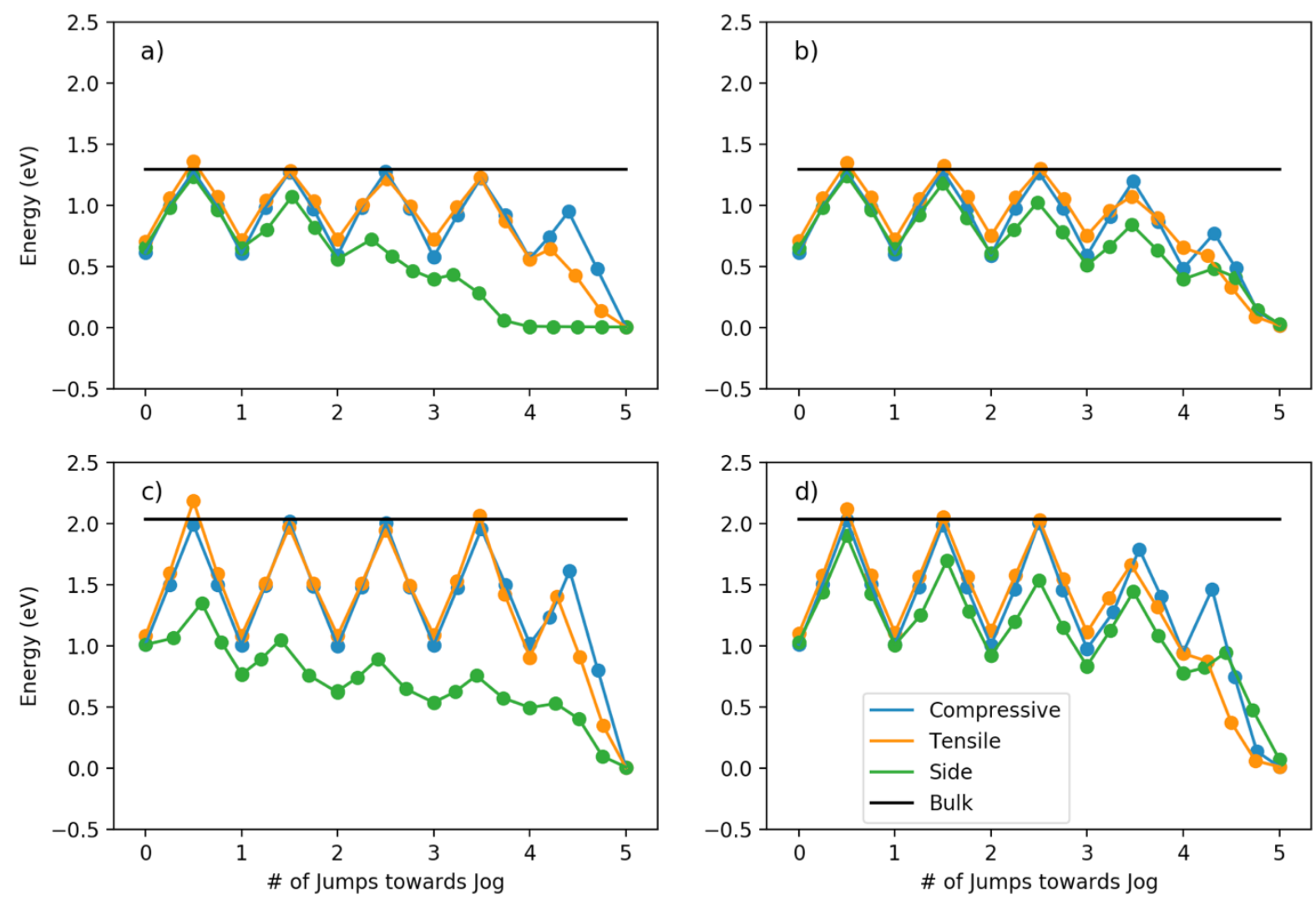

Figure 7: Results for vacancy energetics obtained by the nudged-elastic-band method for extended jogs in a) $\mathrm{Al}$ and c) $\mathrm{Cu}$ and constricted jogs in b) $\mathrm{Al}$ and d) $\mathrm{Cu}$. The black line denotes the sum of the vacancy formation and migration energy in the bulk of the material. All energy values are in reference to the simulation cell without a vacancy.

for a mixed dislocation in $\mathrm{BCC} F e$ show that there is an angular dependence of the near core vacancy barriers for dislocation climb, which is also shown here for the FCC edge dislocation systems. Future work would be warranted to investigate this in further detail, in relation to the validity of assumption 5 of the Balluffi model. However, the results shown here suggest that the assumption is reasonable.

\section{Climb Efficiency}

The results in the previous section suggest the assumptions underlying the theoretical model of Balluffi described in Section $\$ 2$ are reasonable for describing climb velocities for straight edge dislocations in $\mathrm{Al}$ and $\mathrm{Cu}$. In this section, the model is thus used to compute climb efficiencies for these edge dislocations using the values for the relevant parameters obtained in the calculated results presented above. Specifically, the jog-pair formation energy as a function of size can be input into Eq. (10) through the term $E_{\text {jogpair }}\left(N_{\text {vacs }}\right)$, where we will use the values taken from the simulated annealing shown in Fig. 3 The maximum value of Eq. 10 at a fixed temperature and supersaturation will be used 


\begin{tabular}{|c|c|c|c|c|}
\hline Material & $T_{m}(K)$ & $\mathrm{b}(\AA)$ & $\rho\left(\mathrm{m}^{-2}\right)$ & $E_{b}(\mathrm{eV})$ \\
\hline $\mathrm{Al}$ & 926 & 2.86 & $10^{12}$ & 1.296 \\
\hline $\mathrm{Cu}$ & 1353 & 2.57 & $10^{12}$ & 2.037 \\
\hline
\end{tabular}

Table 2: Values for the parameters for $\mathrm{Al}$ and $\mathrm{Cu}$ used in the Baluffi model to compute climb efficiencies as a function of temperature and vacancy supersaturation. The melting temperature is from the potential [37], and $\rho$ is chosen as a realistic order of magnitude value in order to evaluate $R$ in Eq. 5

to find $\lambda$ using Eq. (3). This value will need to be compared with $Z$, calculated through Eq. (2). This requires an evaluation of $\Delta W_{s m}$, which will be taken as $0.2 E_{b}$ as it has been shown that the self-migration energy of a vacancy in the core of an edge dislocation in FCC metals $\left(E_{c}\right)$ is about $0.8 E_{b}$ [43, 44]. The ratio of $Z$ and $\lambda$ allows for the evaluation of the dislocation climb velocity using Eq. (7). Once the climb velocity is evaluated, the climb efficiency is accessible through Eq. (8). Values for the required parameters including the calculated $E_{b}$ are given in Table 2 Climb efficiencies are calculated up to the melting temperature as this is the limit of thermodynamic stability of the solid. Diffusivities are not included because they cancel out in the expressions for climb efficiencies due to the form of Eq. (8). Energetics related to vacancy concentration are similarly not included because they also cancel out in Eq. (8) and only the ratio of the given concentration to the equilibrium concentration matters in Eq. (4).

The resulting values for climb efficiency are plotted as a function of homologous temperature and vacancy supersaturation in Fig. 8. The plots only show values where $R>\lambda$, as this is the limit of the application of the model and it is assumed that when that condition is not satisfied that climb is negligible [1]. The results for both materials feature a sharp transition from a regime of very low climb efficiencies (attachment limited kinetics) at low temperatures and supersaturations, to one of high efficiencies (diffusion limited kinetics) at high temperatures and supersaturations. The sharp transition between these regimes can be interpreted to occur at values of temperature or driving force where the barriers to jog-pair formation becomes negligible relative to the thermal energy. In Fig. 89), we include with the solid point a result from the multiscale simulation study of Baker and Curtin [25], where they find an edge dislocation in Al to become a fully efficient sink at a supersaturation around 145 at a temperature of $600 \mathrm{~K}$. Even though a different potential was used, the results of Baker and Curtin are near our predictions of where the transition to a high climb efficiency at the same temperature and supersaturation conditions occurs. While they did apply a large climb stress (around $1 \mathrm{GPa}$, while keeping the hydrostatic stress equal to zero), our results are consistent with theirs in the sense that we find that it takes a similar number of vacancies to form a jog-pair (4 in our study, 4 in [25]) with a similar potential energy cost (at 4 vacancies, our jog-pair formation energy is around $1.1 \mathrm{eV}$, and an estimate of the summation of the first 4 points in Fig. 4 in [25] leads to $1.2 \mathrm{eV}$ ). It would be of interest for future work to employ the multiscale methodology to other regimes of temperature and supersaturation to investigate whether the transition between low and high climb efficiencies matches the predictions from the present approach.

The results in Fig. 8 show that $\mathrm{Al}$ has a wider range of homologous temperatures and supersaturations where the efficiencies are considerably higher than for $\mathrm{Cu}$. This qualitatively agrees with experimental observations [4, 5] 

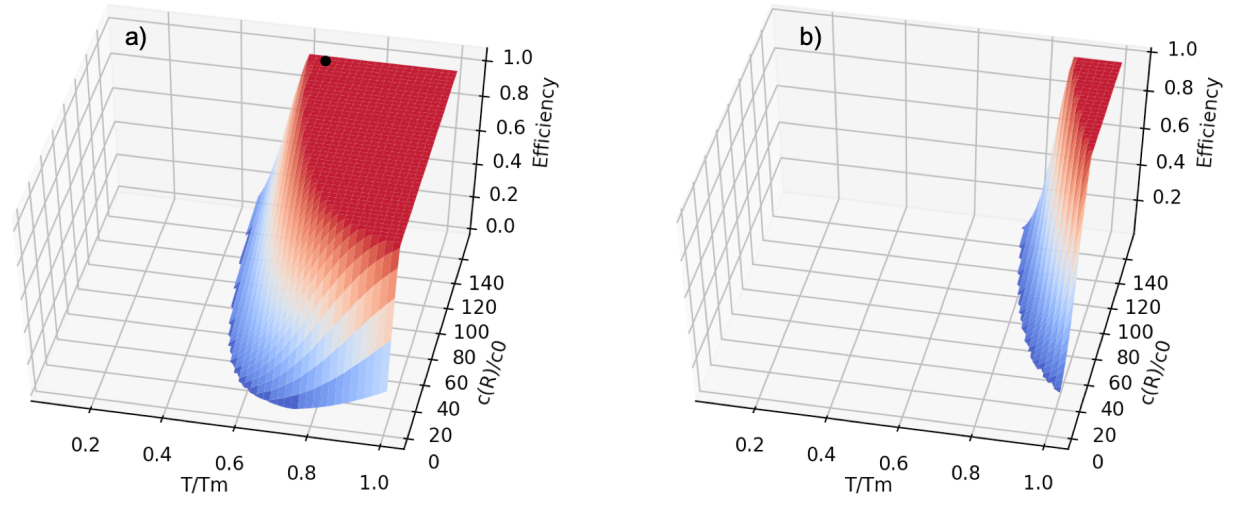

Figure 8: Plots of edge dislocation climb efficiencies of a) $\mathrm{Al}$ and b) $\mathrm{Cu}$ as a function of homologous temperature and ratio of increased concentration. The jog-pair formation energy values were taken from the annealed pathways. The black dot in a) represents a $\frac{c(R)}{c_{0}}$ ratio of 146 and a climb efficiency of 1 at a temperature of $600 \mathrm{~K}$, which is meant to mimic the result in [25].

indicating lower climb efficiencies in systems with lower stacking fault energies. The results are also plotted as climb efficiencies as a function of driving force in Fig. 9 using Eq. (4). These plots show that at a high enough driving force, even a low SFE material can become climb efficient, consistent with experimental conclusions [6, 10]. This suggests that for design of alloys that are resistant to creep, a material with a low-stacking fault energy is preferred, in agreement with Yu and Wang [23], in order to minimize effects due to dislocation climb. Additionally, in the design of materials where void nucleation in irradiated materials should be avoided (such as in Gu et al. [22]), a highstacking fault energy is preferred to lower the jog-pair formation energy, thereby increasing the number of vacancy sinks. The results also demonstrate the limits to the assumption that dislocation climb in a material can be described by either a purely diffusion-limited or attachment-limited model, given that the appropriate behavior can change over time if the supersaturation or temperature are evolving. For such cases, mesoscale models capable of describing mixed attachment and diffusion contributions to climb velocities are required, and for such models atomistic simulations like those employed in this study can be used to provide the necessary material parameters.

It is worth noting that the formalism presented in this section neglects the effects of applied stresses and internal stresses due to neighboring dislocations on the vacancy flux and climb rate, and thus it is appropriate only in the limits where the driving force for climb associate with vacancy supersaturation is dominant. We note that in addition to changing the driving forces for climb, these applied and internal stresses can also affect the factors governing climb efficiency. For example, compressive (tensile) stress along the dislocation glide direction is likely to decrease (increase) the jog-pair formation energy and thus affect the values of the driving force where there is a transition in the climb efficiency. These stresses could also affect diffusion towards and along the dislocation [47], considering either vacancy or interstitial mechanisms [2], and thus affect the mean-free path defined in the Ballufi model. An additional 

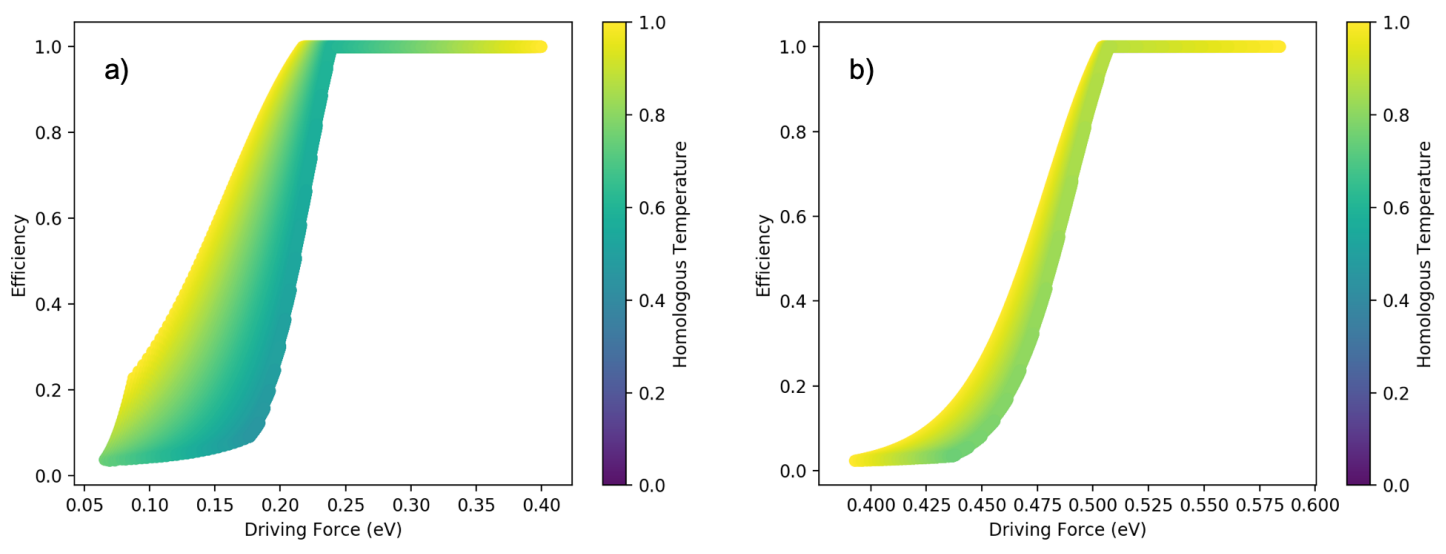

Figure 9: Plots of edge dislocation climb efficiencies of a) $\mathrm{Al}$ and b) $\mathrm{Cu}$ as a function of driving force. The shading represents homologous temperature.

effect is associated with an Escaig stress, which would change the dissociation distance between partial dislocations in the core, with an associated effect on jog-pair formation energy as described above and in the Supplemental Document. Overall, further work would be needed to fully characterize these effects on the climb efficiencies to fully understand the effects of applied and internal stresses on dislocation climb efficiency, and for such studies atomistic kMC models that incorporate stress-dependent values of the different vacancy hops to and from the jogs and straight dislocation segments would be required.

\section{Summary}

Atomistic calculations based on EAM potential models for FCC Al and $\mathrm{Cu}$ have been employed in studies of jog formation energies and vacancy migration energies near jogs, for the purpose of investigating kinetics of dislocation

climb in these representative high and low stacking fault metals. The atomistic results identify low-energy jog-pair structures with varying amounts of absorbed vacancies using simulated thermal annealing. Calculations of energy barriers between different jog-pair structures revealed the existence of a complex kinetic pathway for jog-pair formation in $\mathrm{Cu}$, which likely exists for other low SFE materials more generally. The calculations further demonstrate that there is a tendency for vacancy migration barriers to decrease when moving from the bulk to a jog. The atomistic calculations are used to derive parameters in the theoretical model of climb velocities due to Balluffi [1], to compute climb efficiencies of $\mathrm{Al}$ and $\mathrm{Cu}$ across varying temperature and vacancy supersaturations. The higher SFE $\mathrm{Al}$ system was found to display higher climb efficiencies than $\mathrm{Cu}$ over a wide range of temperatures and supersaturation, in agreement with experimental observations that higher SFE materials show higher efficiencies for climb [2, 4-6]. The effect of applied stress on climb efficiency was not pursued here, but can be incorporated in the presented method by calculating the relevant vacancy and jog-pair energetics under the desired stress state. The atomistic inputs derived 
here to calculate climb efficiency are also necessary for describing mixed kinetic regimes in mesoscale simulations of dislocation climb. The results thus demonstrate how atomistic simulations can be used to improve the generality and accuracy of mesoscale models of material response under creep, annealing, and irradiation conditions.

\section{Acknowledgements}

The authors would like to thank Dr. Emmanuel Clouet for fruitful discussions about jog-pair formation energies. The authors gratefully acknowledge funding from the U.S. Office of Naval Research under grant No. N00014-17-12283. This work made use of computational resources provided by the Extreme Science and Engineering Discovery Environment (XSEDE), which is supported by National Science Foundation under Grant No. ACI-1053575, as well as use of the Savio computational cluster resource provided by the Berkeley Research Computing program at the University of California, Berkeley (supported by the UC Berkeley Chancellor, Vice Chancellor for Research, and Chief Information Officer). A.A. acknowledges a fellowship through the National Science Foundation Graduate Research Fellowship Program (Grant No. DGE 1752814).

\section{References}

[1] R. W. Balluffi, Mechanisms of dislocation climb, physica status solidi (b) 31 (2) (1969) 443-463. doi:10. $1002 /$ pssb. 19690310202

[2] D. Caillard, J.-L. Martin, Thermally activated mechanisms in crystal plasticity, Vol. 8, Elsevier, 2003.

[3] P. M. Anderson, J. P. Hirth, J. Lothe, Theory of dislocations, Cambridge University Press, 2017.

[4] P. S. Dobson, P. J. Goodhew, R. E. Smallman, Climb kinetics of dislocation loops in aluminium, Philosophical Magazine 16 (139) (1967) 9-22. doi:10.1080/14786436708229253

${ }_{370}$ [5] I. A. Johnston, P. S. Dobson, R. E. Smallman, The role of jog nucleation and propagation in the annealing of faulting loops in low stacking fault energy metals, Crystal Lattice Defects 2 (1971) 133-139.

[6] D. N. Seidman, R. W. Balluffi, Dislocations as source and sinks for point defects in metals, in: Lattice defects and their interactions, Gordon and Breach Science Publishers, 1967, pp. 911-960.

[7] J. Silcox, M. J. Whelan, Direct observations of the annealing of prismatic dislocation loops and of climb of dislocations in quenched aluminium, Philosophical Magazine 5 (49) (1960) 1-23. doi:10.1080/ 14786436008241196

[8] C. A. Johnson, The growth of prismatic dislocation loops during annealing, Philosophical Magazine 5 (60) (1960) 1255-1265. doi:10.1080/14786436008238338 
[9] T. D. Swinburne, K. Arakawa, H. Mori, H. Yasuda, M. Isshiki, K. Mimura, M. Uchikoshi, S. L. Dudarev, Fast,

[10] D. N. Seidman, R. W. Balluffi, On the Efficiency of Dislocation Climb in Gold, Physica Status Solidi (B) 17 (2) (1966) 531-541. doi:10.1002/pssb.19660170208

[11] E. Martínez, B. P. Uberuaga, Mobility and coalescence of stacking fault tetrahedra in Cu, Scientific Reports 5 (V) (2015) 1-5. doi:10.1038/srep09084.

[12] D. Mordehai, E. Clouet, M. Fivel, M. Verdier, Introducing dislocation climb by bulk diffusion in discrete dislocation dynamics, Philosophical Magazine 88 (6) (2008) 899-925. doi : 10.1080/14786430801992850.

[13] B. Bakó, E. Clouet, L. M. Dupuy, M. Blétry, Dislocation dynamics simulations with climb: kinetics of dislocation ㄴ loop coarsening controlled by bulk diffusion, Philosophical Magazine 91 (23) (2011) 3173-3191. doi:10. $1080 / 14786435.2011 .573815$

[14] S. M. Keralavarma, T. Cagin, A. Arsenlis, A. A. Benzerga, Power-law creep from discrete dislocation dynamics, Physical Review Letters 109 (26) (2012) 265504. doi:10.1103/PhysRevLett.109.265504.

[15] Y. Gao, Z. Zhuang, Z. L. Liu, X. C. You, X. C. Zhao, Z. H. Zhang, Investigations of pipe-diffusion-based dislocation climb by discrete dislocation dynamics, International Journal of Plasticity 27 (7) (2011) 1055-1071. doi:10.1016/j.ijplas.2010.11.003

[16] X. Niu, T. Luo, J. Lu, Y. Xiang, Dislocation climb models from atomistic scheme to dislocation dynamics, Journal of the Mechanics and Physics of Solids 99 (2017) 242-258. doi:10.1016/j.jmps.2016.11.012.

[17] C. Ayas, J. A. W. Van Dommelen, V. S. Deshpande, Climb-enabled discrete dislocation plasticity, Journal of the Mechanics and Physics of Solids 62 (2014) 113-136. doi:10.1016/j.jmps.2013.09.019

[18] J. H. Ke, A. Boyne, Y. Wang, C. R. Kao, Phase field microelasticity model of dislocation climb: Methodology and applications, Acta Materialia 79 (2014) 396-410. doi:10.1016/j . actamat.2014.07.003.

[19] P. A. Geslin, B. Appolaire, A. Finel, A phase field model for dislocation climb, Applied Physics Letters 104 (1) (2014) 011903. doi:10.1063/1.4860999

[20] P. A. Geslin, B. Appolaire, A. Finel, Multiscale theory of dislocation climb, Physical Review Letters 115 (26) 
[21] P. Liu, S. Zheng, K. Chen, X. Wang, B. Yan, P. Zhang, S.-Q. Shi, Point defect sink strength of low-angle tilt grain boundaries: A phase field dislocation climb model, International Journal of Plasticity 119 (2019) 188-199. doi:10.1016/J.IJPLAS.2019.03.008

[22] Y. Gu, Y. Xiang, D. J. Srolovitz, J. A. El-Awady, Self-healing of low angle grain boundaries by vacancy diffusion and dislocation climb, Scripta Materialia 155 (2018) 155-159. doi:10.1016/j.scriptamat.2018.06.035

[23] X. X. Yu, C. Y. Wang, The effect of alloying elements on the dislocation climbing velocity in Ni: A first-principles study, Acta Materialia 57 (19) (2009) 5914-5920. doi:10.1016/j . actamat.2009.08.019.

[24] S. Sarkar, J. Li, W. T. Cox, E. Bitzek, T. J. Lenosky, Y. Wang, Finding activation pathway of coupled displacivediffusional defect processes in atomistics: Dislocation climb in fcc copper, Physical Review B 86 (2012) 014115. doi:10.1103/PhysRevB.86.014115.

[25] K. L. Baker, W. A. Curtin, Multiscale diffusion method for simulations of long-time defect evolution with application to dislocation climb, Journal of the Mechanics and Physics of Solids 92 (2016) 297-312. doi: $10.1016 / j \cdot j m p s .2016 .04 .006$

[26] T. T. Lau, X. Lin, S. Yip, K. J. Van Vliet, Atomistic examination of the unit processes and vacancy-dislocation interaction in dislocation climb, Scripta Materialia 60 (6) (2009) 399-402. doi:10.1016/j.scriptamat. 2008.11 .019

[27] M. Kabir, T. T. Lau, D. Rodney, S. Yip, K. J. Van Vliet, Predicting dislocation climb and creep from explicit atomistic details, Physical Review Letters 105 (9) (2010) 095501. doi:10.1103/PhysRevLett.105.095501.

[28] E. Clouet, Predicting dislocation climb: Classical modeling versus atomistic simulations, Physical Review B Condensed Matter and Materials Physics 84 (9) (2011) 092106. doi:10.1103/PhysRevB.84.092106.

[29] G. Henkelman, H. Jónsson, Improved tangent estimate in the nudged elastic band method for finding minimum energy paths and saddle points, Journal of Chemical Physics 113 (22) (2000) 9978-9985. doi:10.1063/1. 1323224

[30] G. Henkelman, B. P. Uberuaga, H. Jónsson, Climbing image nudged elastic band method for finding saddle points and minimum energy paths, Journal of Chemical Physics 113 (22) (2000) 9901-9904. doi:10.1063/1. 1329672 .

[31] E. Clouet, L. Ventelon, F. Willaime, Dislocation core energies and core fields from first principles, Physical Review Letters 102 (5) (2009) 055502. doi:10.1103/PhysRevLett.102.055502 
[32] E. Clouet, Babel package. URL http://emmanuel.clouet.free.fr/Programs/Babel/

[33] E. Clouet, Étude par Simulations Numériques de la Plasticité dans les Métaux (2013).

[34] A. N. Stroh, Dislocations and Cracks in Anisotropic Elasticity, Journal of Theoretical Experimental and Applied Physics 330 (1958) 625-646. doi:10.1080/14786435808565804

[35] A. N. Stroh, Steady State Problems in Anisotropic Elasticity, Journal of Mathematics and Physics 41 (1-4) (1962) 77-103. doi:10.1002/sapm196241177

[36] W. Cai, V. V. Bulatob, J. Chang, J. Li, S. Yip, Periodic image effects in dislocation modelling, Philosophical Magazine 83 (5) (2003) 539-567. doi:10.1080/0141861021000051109

[37] M. I. Mendelev, M. J. Kramer, C. A. Becker, M. Asta, Analysis of semi-empirical interatomic potentials appropriate for simulation of crystalline and liquid $\mathrm{Al}$ and $\mathrm{Cu}$, Philosophical Magazine 88 (12) (2008) 1723-1750. doi:10.1080/14786430802206482

[38] E. Bitzek, P. Koskinen, F. Gähler, M. Moseler, P. Gumbsch, Structural relaxation made simple, Physical Review Letters 97 (17) (2006) 170201. doi:10.1103/PhysRevLett.97.170201.

[39] S. Plimpton, Fast Parallel Algorithms for Short-Range Molecular Dynamics, Journal of Computational Physics 117 (1) (1995) 1-19. doi:10.1006/JCPH.1995.1039.

[40] A. Stukowski, Visualization and analysis of atomistic simulation data with OVITO-the Open Visualization Tool, Modelling and Simulation in Materials Science and Engineering 18 (1) (2010) 015012. doi:10.1088/ $0965-0393 / 18 / 1 / 015012$

[41] A. Stukowski, Structure identification methods for atomistic simulations of crystalline materials, Modelling and a Simulation in Materials Science and Engineering 20 (4) (2012) 045021. doi:10.1088/0965-0393/20/4/ 045021

[42] M. Landeiro Dos Reis, L. Proville, M. Sauzay, Modeling the climb-assisted glide of edge dislocations through a random distribution of nanosized vacancy clusters, Physical Review Materials 2 (2018) 93604. doi:10.1103/ PhysRevMaterials.2.093604.

[43] G. P. Purja Pun, Y. Mishin, A molecular dynamics study of self-diffusion in the cores of screw and edge dislocations in aluminum, Acta Materialia 57 (18) (2009) 5531-5542. doi:10.1016/j.actamat.2009.07.048. 
[44] J. Huang, M. Meyer, V. Pontikis, Migration of point defects along a dissociated edge dislocation in copper: a molecular dynamics study of pipe diffusion, Philosophical Magazine A 63 (6) (1991) 1149-1165. doi:10. $1080 / 01418619108205574$

[45] J. Grilhé, M. Boisson, K. Seshan, R. J. Gaboriaud, Climb model of extended dislocations in f.c.c. metals, Philosophical Magazine 36 (4) (1977) 923-930. doi:10.1080/14786437708239767.

[46] P. B. Hirsch, Extended Jogs in Dislocations in Face-centred Cubic Metals, Philosophical Magazine 7 (73) (1962) 67-93. doi:10.1080/14786436208201859.

[47] D. Carpentier, T. Jourdan, Y. Le Bouar, M.-C. Marinica, Effect of saddle point anisotropy of point defects on - their absorption by dislocations and cavities, Acta Materialia 136 (2017) 323-334. doi:10.1016/J. ACTAMAT . 2017.07 .013 